\title{
Novel Multimode Interference Liquid Level Sensors
}

\author{
J. E. Antonio-Lopez ${ }^{\mathrm{a}, *}$, M. Torres-Cisneros ${ }^{\mathrm{b}, \mathrm{d}}$, J. A. Arredondo-Lucio ${ }^{\mathrm{c}}$, J. J. Sanchez-Mondragon ${ }^{\mathrm{a}}$, \\ P. LiKamWa ${ }^{d}$, and D. A. May-Arrioja ${ }^{c}$ \\ ${ }^{a}$ Photonics and Optical Physics Laboratory, Optics Department, INAOE \\ Apdo. Postal 51 y 216, Tonantzintla, Puebla 72000, México \\ ${ }^{b}$ NanoBioPhotonics Group, DICIS, Universidad de Guanajuato \\ Salamanca, Gto.36885 México \\ 'Depto. de Ingeniería Electrónica, UAM Reynosa Rodhe, Universidad Autónoma de Tamaulipas \\ Carr. Reynosa-San Fernando s/n, 88779 Reynosa, Tamaulipas, México \\ ${ }^{\mathrm{d}}$ CREOL, The College of Optics and Photonics, University of Central Florida \\ Orlando, FL 32816-2700, USA.
}

\begin{abstract}
A novel all-fiber Multimode Interference (MMI) liquid level sensor is proposed and demonstrated. We show that MMI effects can be effectively applied for multiplexed liquid level sensing, and by selecting an adequate fiber, discrete and continuous level sensing is feasible. Using a standard 105/125 multimode fiber a simple discrete level sensor was fabricated, which can also can also discriminate the refractive index of the liquid. When a specialty fiber, know as NoCore fiber is used, both continuous and discrete level sensing can be achieved. We can also modify at will the continuous level range by increasing the No-core fiber by the appropriate length, while retaining the ability to determine the refractive index of the liquid during the level measurement. The MMI liquid level sensors are not only inexpensive, but their fabrication is quite simple.
\end{abstract}

Keywords: Optical Fiber Sensor, Multimode Interference, MMI, Fiber Sensor, Sensor, Liquid Level Sensor.

\section{INTRODUCTION}

Optical fiber sensors (OFS) have been the subject of research for several years. They have many attractive features that are superior to electrical systems. Some of the key advantages include low power consumption, ability to withstand corrosive and high temperature environments, immunity from electromagnetic interference, large distance between signal generation/detection, and high sensitivity. Liquid level sensing of flammable fluids is one particular application where OFS are well suited since it does not requires electrical signals. In general we can have two types of level sensor: continuous and discrete. Different configurations have been proposed for discrete optical level sensors such as, exposed fiber gratings [1], and special fiber tips which rely on intensity variations as the liquid level makes contact with the sensing region $[2,5]$. In the case of special fiber tips they operate on frustrated total internal reflection in fibers which could be obtained through angled or retroreflecting tips [2], conical/tapered tips [3], special fiber tip design [4], or the attachment of reflecting elements as prisms [5]. In all cases the need to have a special fiber tip requires either very special fiber treatment or the attachment of an external component, which increases the sensor complexity and cost.

Recently, we have demonstrated the advantages of fiber based MMI devices for different laser applications [6]. The advantages of MMI devices are that they exhibit a pass-band filter response, and they only require splicing a multimode fiber (MMF) between two single mode fibers (SMF). In this work, we propose and demonstrate a novel, yet quite simple, fiber optic level sensor based on MMI effects. The first scheme uses a 105/125 standard multimode fiber (MMF), in which the intensity of the filter is reduced when the liquid makes contact with the tip of the fiber. The MMI effect allows us to have multiplexed operation by simple changing the length of the MMF section. Another scheme uses a special fiber know as No-core fiber, a $105 \mu \mathrm{m}$ diameter MMF without clad, and we are able to shift the spectral response of the filter when the liquid level is increased around the No-Core fiber. In this case, multiplexed operation, discrete and continuous level sensing is feasible. The fabrication of the sensors is straightforward and with a minimum cost, which is very promising for several applications.

*Email: enriquelme@inaoep.mx; Phone: +52 (222) 247-2011 Ext. 9307; Fax: +52 (222) 247-2940; www.inaoep.mx

2nd Workshop on Specialty Optical Fibers and Their Applications (WSOF-2),

edited by Juan Hernández-Cordero, Ismael Torres-Gómez, Alexis Méndez, Proc. of SPIE

Vol. 7839, 78391V · C 2010 SPIE · CCC code: 0277-786X/10/\$18 - doi: 10.1117/12.868256

Proc. of SPIE Vol. 7839 78391V-1 


\section{MULTIMODE INTERFERENCE LIQUID LEVEL SENSOR.}

An schematic of the MMI liquid level sensors is shown in Fig. 1. The key component required is a MMF, which for this application is spliced to only one single mode fiber. For the 105/125 sensor, after splicing the right MMF length the sensor is ready for testing. In the case of the No-Core based MMI sensor a $200 \mathrm{~nm}$ gold layer is evaporated on the fiber facet to act as a mirror.

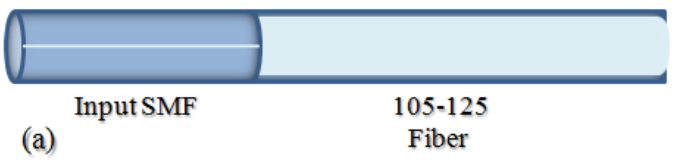

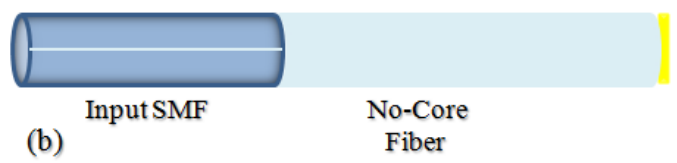

Figure 1. Schematic of MMI Liquid Level Sensors.

Light coupled to the MMF through the SMF will form images of the input field along the MMF. By cleaving the MMF at a precise length, we can decide the peak wavelength of our filter, this can be easily calculated for the fourth image by,

$$
\lambda_{0}=\frac{4 n_{M M F} D_{M M F}^{2}}{L} .
$$

Here $n_{M M F}$ and $D_{M M F}$ correspond respectively to the refractive index and diameter of the MMF and $\lambda_{0}$ as the free space wavelength. Depending on the MMF we can have different type of liquid level sensor. In the case of 105/125 MMF, as shown in Fig. 1 (left), the image formed at the facet of the MMF fiber will be reflected based on the Fresnel reflection of the fiber-air interface. The image formed at the facet will be then reflected, and coupled back to the SMF. Any change due to a liquid at the fiber-air interface will reduce the reflected image intensity and thus providing a simple way to sense the liquid level. As shown in Eq. (1), by changing the length of the MMF we can change the peak wavelength response, and therefore a multiplexed operation can be easily achieved. When using the No-core fiber the operation mechanism is slightly different. As shown in Eq. (1), a way to modify the peak wavelength is by changing the MMF diameter. The NoCore fiber does not have a cladding, therefore when a liquid with a refractive index lower than that of the core touches the fiber, the effective width of the fundamental mode is increased and thus the peak wavelength should shift to longer wavelengths. Since the detection takes place around the fiber, we can then evaporate a gold mirror on the fiber facet which improves the intensity of the optical signal. Additionally, since the liquid is slowly covering the No-Core fiber the wavelength shift should be linear, which operates as a continuous level sensor.

\section{DISTRIBUTED MMI LIQUID LEVEL SENSOR USING 105-125 FIBER}

The response of the MMI liquid level sensor was tested using the setup shown in Fig. 2. Light from an Agilent tunable laser (1460 to $1580 \mathrm{~nm})$ was coupled in the port 1 of an optical circulator, the output from port 2 is connected to the input of a $1 \times 2$ splitter ( $3 \mathrm{~dB}$ splitting ratio) and each one of the output is spliced to a No-Core Fiber segment. The image formed at the facet of the MMF is reflected and coupled back to the SMF, and we measured the response through the output port 3. Light was measured using an InGaAs photodetector connected to a Keithley digital multimeter (DMM). The setup was fully controlled through GPIB ports using LabVIEW.

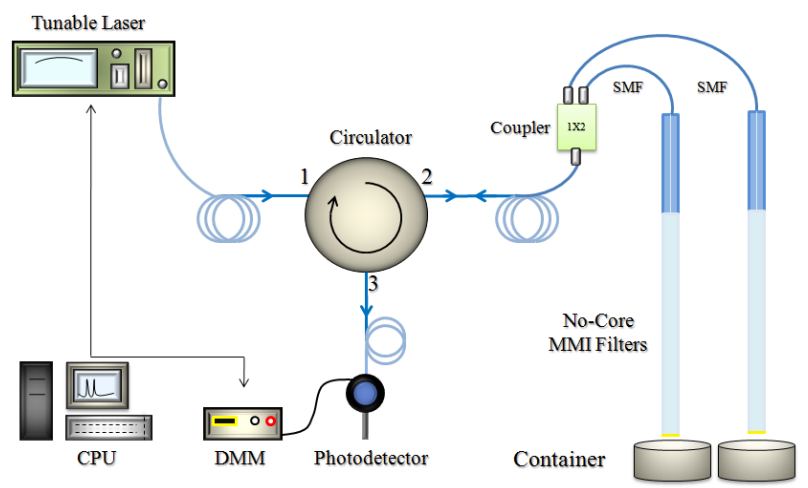

Figure 2. Experimental setup for the liquid level sensor for distributed sensing. 
In the experiments the 105/125 MMF was cleaved at two different lengths, which corresponds to peak wavelengths in air of 1493 and $1560 \mathrm{~nm}$. The response is acquired by making wavelength scans in air, and after the liquid level is raised (in a single or both containers) we take another scan. As shown in Fig. 3(a), the response of a single MMI sensor in air has a pass-band filter like response. However, when the water level is raised and touches the fiber tip, the intensity drops about $5 \mathrm{~dB}$. By using both fibers, we can monitor the level on two different containers simultaneously. Fig. 4(b) shows the response of both fibers in air, and also when both fibers tips touch the water. The peak wavelength corresponds to a container and thus multiplexed monitoring is easily achieved. Fig. 4(c) shows the response when one fiber is in air and the other one in water, and viceversa. And finally, Fig. 4 (d), shows the response when one fiber is in water and the other one in isopropanol, and viceversa. Besides the multiplexing properties, the difference in intensity resulting from the different refractive index of the liquid can also tell us which kind of liquid we are detecting.
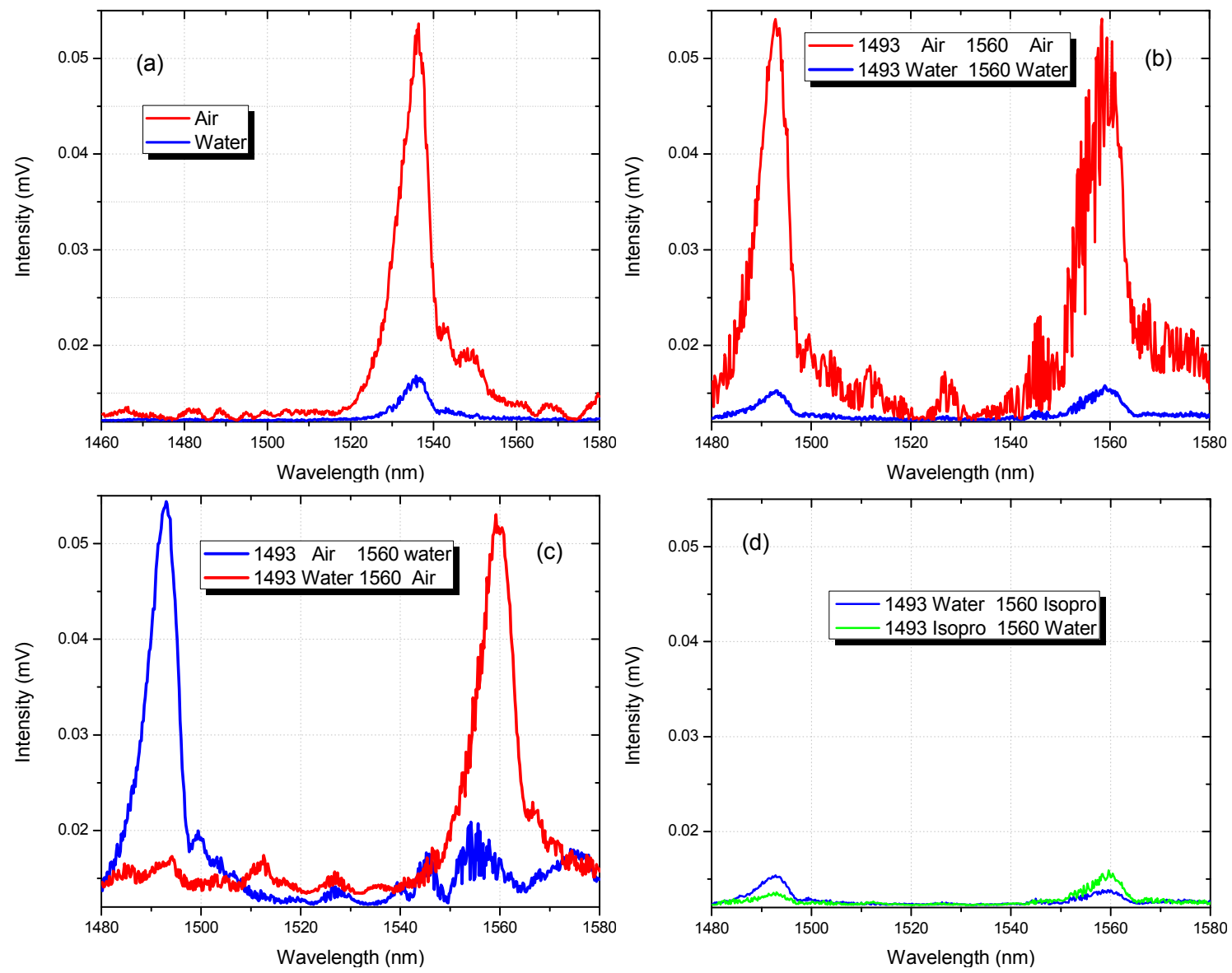

Figure 3. (a) Liquid level sensor response with the fiber in air and water, (b-d) Liquid level sensor response for different combinations of air and liquid, and also two different liquids .

\section{DISTRIBUTED MMI LIQUID LEVEL SENSOR USING NO-CORE FIBER}

The MMI based liquid level sensors based on No-Core fiber are fabricated in a similar way. The No-Core fibers are cleaved to provide peak wavelengths at 1484 and $1524 \mathrm{~nm}$, as shown in Fig. 4 (black line). After cleaving, a $200 \mathrm{~nm}$ gold mirror is carefully evaporated on the fiber facet to act as a mirror to prevent losses due to reduction of Fresnel reflection. As explained before, when the liquid makes contact with the fiber, the length of the No-Core fiber in contact with the liquid can be seen as having a slight different diameter. Therefore, the MMI effect is now calculated taken the accumulated phase of this section plus the section that has no liquid. The net result is that the peak wavelength is shifted to longer wavelengths, as shown in Fig. 4 (left for the MMI with $1484 \mathrm{~nm}$ peak wavelength). As the liquid level is increased, the wavelength keeps shifting until the No-Core fiber is completely covered, and the shift stops. A similar situation can be observed for the MMI with $1524 \mathrm{~nm}$ peak wavelength, as shown in Fig. 4 (right). 
The sensor response can be used for continuous liquid level sensing, and the range can be increased by increasing the length of the No-Core fiber and operate the MMI sensor at higher image orders. If discrete operation is required, we can cover the top $50 \mathrm{~mm}$ of the fiber with a low refractive index material, such as PDMS, and have the liquid to interact only the within $10 \mathrm{~mm}$ of length of the No-Core fiber. In this situation, the peak wavelength will shift about $6 \mathrm{~nm}$, which is enough to detect when the liquid makes contact with the fiber. An attenuation of $7.5 \mathrm{~dB}$ is obtained which is good enough for liquid level sensing.
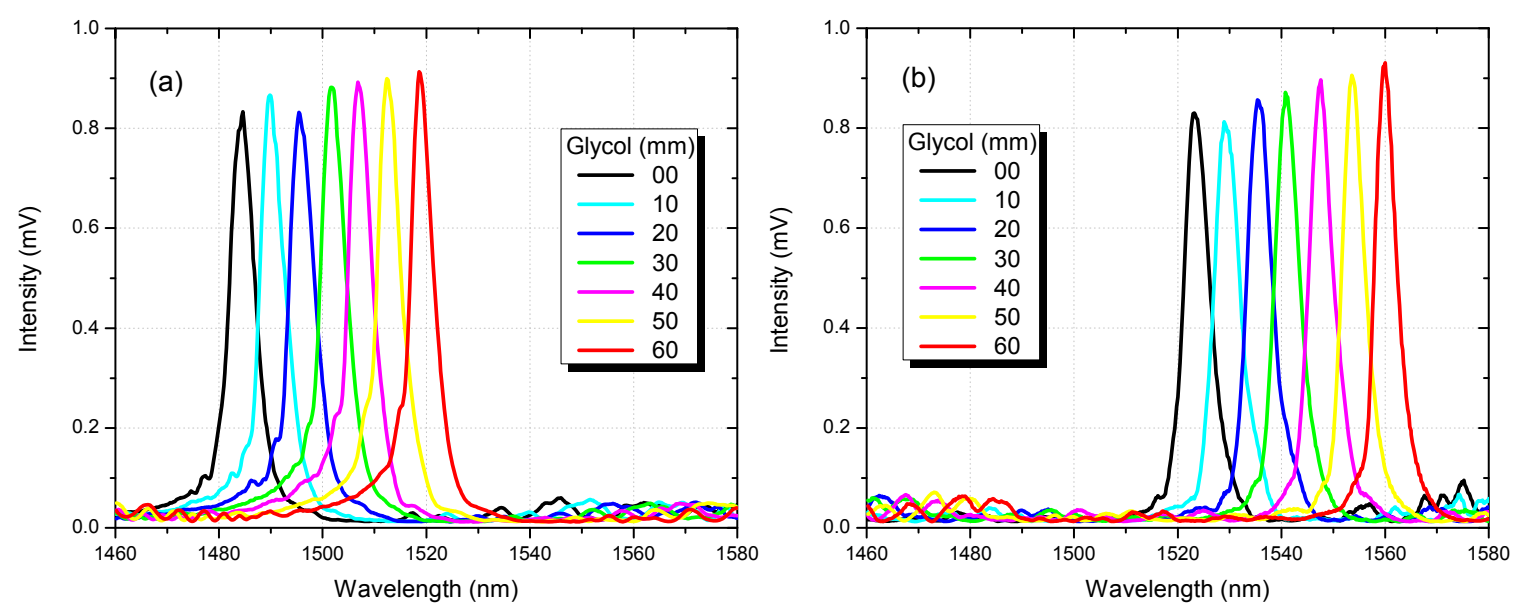

Figure 4. (a-b) Liquid level sensing at two different peak wavelengths using the No-Core fiber.

\section{CONCLUSIONS}

We demonstrated a novel all-fiber liquid level sensor based on multimode interference (MMI). The MMI liquid level sensors are easy to fabricate which results in a cost-effective sensor. Using this principle two kinds of MMI level sensor were fabricated, and even when they operate based on MMI effects to achieve multiplexed operation, the sensing capabilities are quite different. By choosing the right MMF we can also achieve discrete and continuous operation with an easily adjustable range. This kind of sensor are very promising not only for liquid level sensing, but also for refractive index sensing applications since they can easily discriminate the refractive index of the liquid.

\section{REFERENCES}

[1] S. Khaliq, S. W. James, and R. P. Tatam, "Fiber-optic liquid-level sensor using a long period grating," Opt, Lett. 26(16), 1224-1226 (2001).

[2] I. K. Ilev and R. W. Waynant, "All-fiber-optic sensor for liquid level measurement," Rev. Sci. Instrum. 70(5), 25512554 (1991).

[3] L. Ren and Q. Yu, "High accuracy fiber optic level sensor," Proc. SPIE 4920 362-366 (2002).

[4] P. Raatikaine, I. Kassamakov, and M. Luukkala, "Fiber-Optic liquid-level sensor," Sens. Actuators, A A58, 93-97 (1997).

[5] C. Yang S. chen, and G. Yang "Fiber Optic Liquid Level sensor under cryogenic environment," Sens. Actuators, A A94(1), 69-75 (2001).

[6] J. E. Antonio-Lopez, A. Castillo-Guzman, D. A. May-Arrioja, R, Selvas-Aguilar and P. LiKamWa, "Tunable Multimode-interference Bandpass fiber filter," Opt. Lett. 35(3), 324-326 (2010). 